\title{
Extracting automata from neural networks using active learning
}

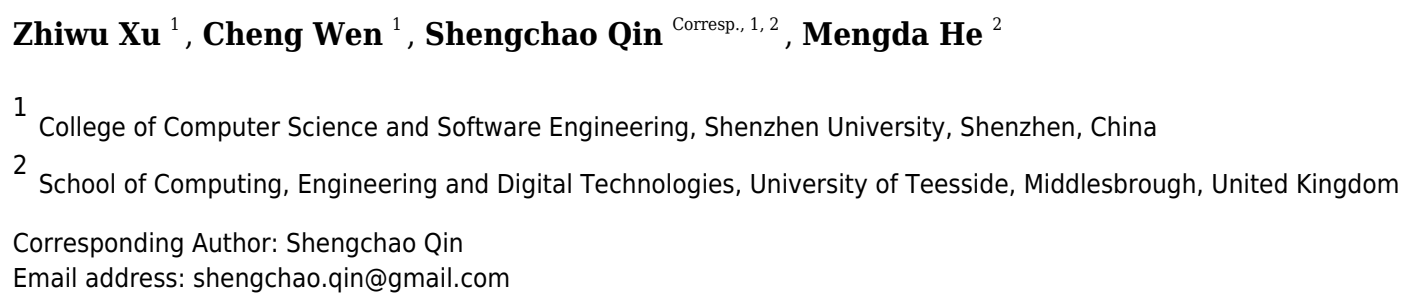

Deep Learning is one of the most advanced forms of Machine Learning. Most modern deep learning models are based on an artificial neural network, and benchmarking studies reveal that neural networks have produced results comparable to and in some cases superior to human experts. However, the generated neural networks are typically regarded as incomprehensible black-box models, which not only limits their applications, but also hinders testing and verifying. In this paper, we present an active learning framework to extract automata from neural network classifiers, which can help users to understand the classifiers. In more detail, we use Angluin's L* algorithm as a learner and the neural network under learning as an oracle, employing abstraction interpretation of the neural network for answering membership and equivalence queries. Our abstraction consists of value, symbol and word abstractions. The factors that may affect the abstraction are also discussed in the paper. We have implemented our approach in a prototype. To evaluate it, we have performed the prototype on a MNIST classifier and have identified that the abstraction with interval number 2 and block size $1 \times 28$ offers the best performance in terms of F1 score. We also have compared our extracted DFA against the DFAs learned via the passive learning algorithms provided in LearnLib and the experimental results show that our DFA gives a better performance on the MNIST dataset. 


\title{
Extracting Automata from Neural Networks Using Active Learning
}

\author{
Zhiwu Xu ${ }^{1}$, Cheng Wen ${ }^{1}$, Shengchao Qin ${ }^{2,1}$, and Mengda $\mathrm{He}^{2}$ \\ ${ }^{1}$ College of Computer Science and Software Engineering, Shenzhen University, \\ Shenzhen, China \\ ${ }^{2}$ School of Computing, Engineering and Digital Technologies, Teesside University, \\ Middlesbrough, United Kingdom
}

Corresponding author:

Shengchao Qin ${ }^{2,1}$

Email address: shengchao.qin@gmail.com

\begin{abstract}
Deep Learning is one of the most advanced forms of Machine Learning. Most modern deep learning models are based on an artificial neural network, and benchmarking studies reveal that neural networks have produced results comparable to and in some cases superior to human experts. However, the generated neural networks are typically regarded as incomprehensible black-box models, which not only limits their applications, but also hinders testing and verifying. In this paper, we present an active learning framework to extract automata from neural network classifiers, which can help users to understand the classifiers. In more detail, we use Angluin's $L^{*}$ algorithm as a learner and the neural network under learning as an oracle, employing abstraction interpretation of the neural network for answering membership and equivalence queries. Our abstraction consists of value, symbol and word abstractions. The factors that may affect the abstraction are also discussed in the paper. We have implemented our approach in a prototype. To evaluate it, we have performed the prototype on a MNIST classifier and have identified that the abstraction with interval number 2 and block size $1 \times 28$ offers the best performance in terms of $F 1$ score. We also have compared our extracted DFA against the DFAs learned via the passive learning algorithms provided in LearnLib and the experimental results show that our DFA gives a better performance on the MNIST dataset.
\end{abstract}

\section{INTRODUCTION}

Deep Learning is one of the most advanced forms of Machine Learning, which has been applied to various fields, including computer vision, speech recognition, natural language processing, audio recognition, social network filtering, machine translation, bioinformatics, drug design and board game programs (Schmidhuber, 2014; Lecun et al., 2015). Most modern deep learning models are based on an artificial neural network, such as deep neural networks (DNN), deep belief networks (DBN), convolutional neural networks $(\mathrm{CNN})$ and recurrent neural networks (RNN). Benchmarking studies reveal that neural networks have produced results comparable to and in some cases superior to human experts.

However, the generated neural networks are typically regarded as incomprehensible black-box models. They are in practice unlikely to generalise exactly to the concept being trained, and what they eventually learn actually is unclear (Omlin and Giles, 2000). The opaqueness of neural networks not only limits their applications, but also hinders testing and verifying. Indeed, several lines of work attempt to glimpse into the black-box networks, especially RNN (Zeng et al., 1993; Sanfeliu and Alquezar, 1994; Tiňo and Šajda, 1995; Omlin and Giles, 1996; Frasconi et al., 1996; Gori et al., 1998; Cechin et al., 2003; Cohen et al., 2017). They induce rules that mimic the blackbox neural networks as closely as possible, by exploring the possible state vectors of networks, which is often practically impossible at present.

Active Learning (Angluin, 1987) can learn finite automata (sets of words) from a minimally adequate teacher, an oracle capable of answering the so-called membership and equivalence queries, which has been successfully applied to numerous practical cases in different domains (Vaandrager, 2017; Aichernig et al., 2018). Recently, Weiss et al. (Weiss et al., 2018) adopted active learning to extract automata from 
neural networks. But the network systems under learning are RNN acceptors on small regular languages: an input at a time is considered as a symbol, and thus a sequence of inputs is a word. Hence, their approach is not suitable to other neural networks in practice such as CNN, since not all neural networks perform on discrete-time symbolic data.

In this paper, we present an active learning framework to extract automata from neural network classifiers, which is inspired by Weiss et al.'s work (Weiss et al., 2018). But different from their work, we consider each input as a word (i.e., a sequence of symbols) rather than a symbol, via abstraction interpretation, which consists of value, symbol and word abstractions. So the system under learning here can be any neural network. Indeed, we assume that we have no idea about the framework of neural networks: we can not access the state-vectors nor we do not know the relations between two consecutive inputs. For simplicity, we focus on network-acceptors, that is, binary neural network classifiers, since multi-class classifiers can be reduced into several binary classifiers ${ }^{1}$.

Abstraction is the key for scaling model learning methods to realistic application (Vaandrager, 2017). So the key idea of our approach is to define an abstraction for the neural network classifier under learning. Our abstraction consists of three layers: (1) value abstraction: each value in an input array is mapped into an integer via partitioning; (2) symbol abstraction: a block of multi-dimensional integer array is abstracted as a symbol; and (3) word abstraction: the whole input array is encoded into a word. We also discuss the factors that may affect the abstraction.

Next, we present how to instantiate the active learning framework on neural networks, in particular the membership and equivalence queries (Vaandrager, 2017). Membership queries can be answered by the neural networks via the word concretization function: we concretise the word that is being queried and then feed the concretised data into the neural network under learning. Equivalence query is more of a challenge, because there is no finite interpretation for neural networks (Weiss et al., 2018). To address this, we use as an abstract model the automaton that is learned passively from some test data in the training dataset and then perform the equivalence query against the abstract model. If no words that separate the hypothesis and the abstract model are found, then the answer for the equivalence query is yes. Note that, when a counterexample is found, it may be not that the hypothesis is incorrect, but rather that the abstract model is not precise enough and needs to be refined.

Finally, we have implemented our approach in Java, wherein we use the library LearnLib (Howar et al., 2012) to implement the active learning framework. To evaluate our approach, we conducted a series of experiments on a classifier for the MNIST dataset, a large database of handwritten digits that is commonly used for training various image processing systems. We first test the measures of the MNIST classifier, namely, safety, conflict, the size of alphabet and the length of words, under the abstractions with different interval numbers (i.e., the number of partitioning) and block sizes, and have identified some suitable abstractions. Secondly, we conduct some experiments to learn DFAs from the MNIST classifier with the suggested abstractions. The results shows that the abstraction with interval number 2 and block size $1 \times 28$ offers the best performance in terms of F1 score. At last, we also conduct the experiments to compare our resulted DFA against the DFAs learned via the passive learning algorithms (see Section 2.4) provided in LearnLib and the MNIST classifier itself. Although worse than the classifier, our DFA gives a better performance than the other DFAs in our experiments. Nevertheless, there are still some limitations for our approach.

In summary, our contributions are as follows:

- We have proposed an MAT framework to extract automata from neural networks, employing abstraction interpretation of the neural network for answering membership and equivalence queries.

- We have conducted several experiments on a MNIST classifier, and the experimental results show that our approach is viable, and the resulted DFA has a better performance in terms of F1 score than the DFAs learned via the passive learning algorithms provided in LearnLib on the MNIST dataset.

The remainder of this paper is organised as follows. Section 2 gives the preliminaries of DFA and active learning. Section 3 describes our approach, followed by the experimental results in Section 4 . Section 5 discusses some limitations of our approach. Section 6 presents the related work, followed by some concluding remarks in Section 7.

${ }^{1}$ This may raise a comparative performance problem, which is not the scope of this paper. 
112• $Q$ is a finite set of states,

${ }_{113} \bullet \Sigma$ is a finite set of input symbols and is called the alphabet,

114- $\delta: Q \times \Sigma \rightarrow Q$ is the transition function,

$115 \bullet q_{0} \in Q$ is the starting state,

116 $F \subseteq Q$ is the set of accepting states.

${ }_{123} \bullet r_{0}=q_{0}$

124• $r_{i+1}=\delta\left(r_{i}, a_{i+1}\right)$, for $i=0, \ldots, n-1$

125• $r_{n} \in F$. length 0 and contains no symbols. following conditions:

A word or string over an alphabet $\Sigma$ is a finite sequence of symbols from $\Sigma$. The length of a word is the number of symbols it contains. Note that a word can be empty: the empty word, denoted as $\varepsilon$, has

Definition 2.2. Let $\mathscr{M}=\left(Q, \Sigma, \delta, q_{0}, F\right)$ be a DFA and $w=a_{1} a_{2} \ldots a_{n}$ be a word of length $n$ over $\Sigma$. The automaton $\mathscr{M}$ accepts the word $w$ if and only if there exists a sequence of states $r_{0}, r_{1}, \ldots, r_{n}$ with the

The set of words recognised by a DFA $\mathscr{M}$, called the language of $\mathscr{M}$, is the following set:

$$
L(\mathscr{M})=\left\{w \in \Sigma^{*} \mid w \text { is accepted by } \mathscr{M}\right\}
$$

\subsection{Active Learning Framework}

Angluin (Angluin, 1987) proposed the first active learning algorithm, the $L^{*}$ algorithm, to learn finite automata from a minimally adequate teacher (MAT) in 1987, and today all the most efficient learning algorithms that are being used follow Angluin's approach. In the following, we briefly introduce the MAT framework.

Finite automata can be learned precisely from a minimally adequate teacher (MAT), that is, an oracle capable of answering the so-called membership and equivalence queries:

- membership queries (MQ): the learner asks whether a given word is accepted by the automaton or not, and the teacher answers with the result.

- equivalence queries (EQ): the learner asks whether a given hypothesis automaton $\mathscr{H}$ is equal to the automaton model $\mathscr{M}$ held by the teacher. The teacher answers yes if this is the case. Otherwise she answers no and supplies a word, the so-called counterexample, on which the hypothesis automaton $\mathscr{H}$ and the automaton model $\mathscr{M}$ disagree. 


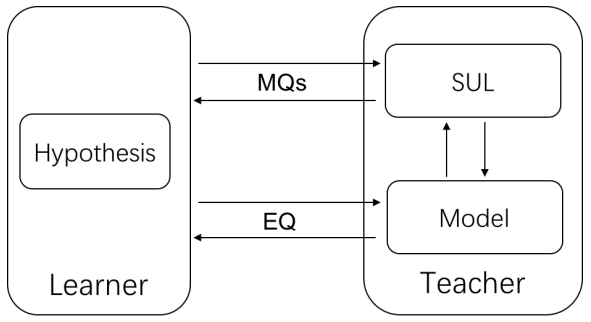

Figure 1. The MAT Framework

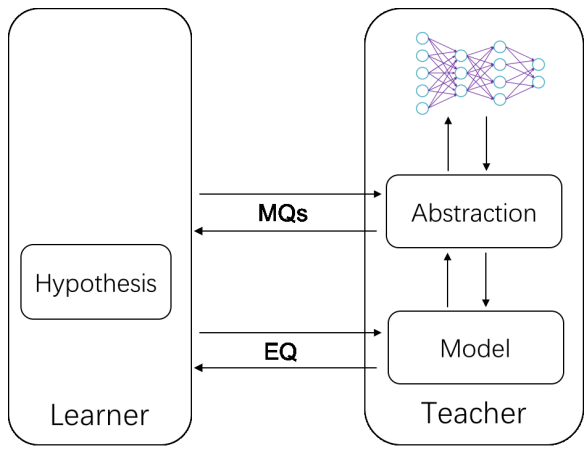

Figure 2. Framework of Our Approach

The MAT framework is shown in Figure 1. Initially, the learner knows the static interface of the system under learning (SUL), that is, the sets of input (i.e., multi-dimensional array for neural networks) and output (i.e., yes or no for recognizers). Then the learner starts to ask a sequence of membership queries and receives the corresponding responses from the teacher. After a "sufficient" number of queries, the learner builds a hypothesis $\mathscr{H}$ from the obtained information, and then sends an equivalence query. If the teacher answers yes, then the hypothesis $\mathscr{H}$ is returned. Otherwise, the learner refines the information with the returned counterexample, and continues on querying.

\subsection{Passive Learning}

Different from active learning, passive learning constructs automata from sets of examples directly. Many approaches in grammatical inference can be described as passive learning. In the paper, we consider the polynomial-time RPNI algorithms provided in the library LearnLib (Howar et al., 2012).

Oncina and García (Oncina and García, 1992) proposed the Regular Positive and Negative Inference (RPNI) algorithm for DFA learning. RPNI starts with a prefix tree acceptor, a tree-like DFA built from the learning examples by taking all the prefixes of the examples as states, and then greedily creates clusters of states (by merging) in order to come up with an automaton that is always consistent with the examples. Two heuristic strategies can be employed in state merging: Evidence Driven State Merging (EDSM) (Cicchello and Kremer, 2002) and Minimum Description Length (MDL) (Adriaans and Jacobs, 2006).

\section{APPROACH}

In this section, we present an active learning framework to extract automata from neural network classifiers. Our framework is shown in Figure 2, which is a classic MAT framework with an abstraction ${ }^{2}$. In a nutshell, we make an abstraction between the learner and the system under learning. When the membership queries are sent, the abstraction maps the abstract words into the concrete ones (i.e., the up arrow in Figure 2), which are then fed into the neural network under learning. When the equivalence queries are sent, the abstraction does the opposite (i.e., the down arrow in Figure 2) and from the abstract words an abstract representation is built for checking. In the following, we explain how to define an abstraction for neural networks and how to instantiate the active learning framework on neural networks.

\subsection{Abstraction}

For simplicity, we focus on network-acceptors, that is, there are only two outputs for the system under learning, and thus we do not need to abstract them. In other words, only the inputs need to be abstracted. Generally, the inputs of neural network classifiers are always multi-dimensional arrays. As mentioned in Section 1, we aim to abstract an input as a word, rather than a symbol. So the aim of the abstraction is to convert multi-dimensional arrays into words and vice-versa.

Just like the serialization of multi-dimensional arrays, a naive and simple solution to the abstraction is to convert the input multi-dimensional array into a 1-dimensional array in row (or column) major order, and then concatenate the string representation of each value in the converted array in order, yielding a

${ }^{2}$ Some papers use the term mapper (Vaandrager, 2017). 


\begin{tabular}{|l|l|l|l|}
\hline 0.7 & 0.4 & 0.6 & 0.8 \\
\hline 0.3 & 0.5 & 0.7 & 0.9 \\
\hline 0.6 & 0.8 & 0.2 & 0.4 \\
\hline 0.7 & 0.1 & 0.3 & 0.5 \\
\hline
\end{tabular}

Figure 3. Example of Simple Abstraction

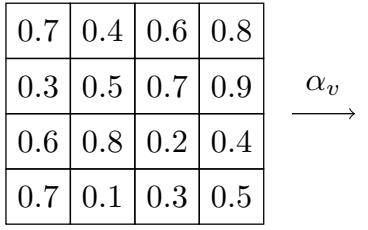

\begin{tabular}{|l|l|l|l|}
\hline 1 & 0 & 1 & 1 \\
\hline 0 & 1 & 1 & 1 \\
\hline 1 & 1 & 0 & 0 \\
\hline 1 & 0 & 0 & 1 \\
\hline
\end{tabular}

Figure 4. Example of Value Abstraction time-consuming. helps reduce the size of alphabet; enables us to reduce the length of word; symbol abstraction are applied. as follows: and $\mathscr{I}$ is split into $I_{0}=[0,0.5)$ and $I_{1}=[0.5,1]$.

$$
\gamma_{v}(i)=I_{i}
$$

where $f_{v}$ is $\alpha_{v}$ or $\beta_{v}$. to say, we define a weak value concretization function: the better. word. For example, Figure 3 shows an array with size $4 \times 4$, on which the simple abstraction is applied. However, there are two problems for this solution: (1) the size of alphabet may be too large, even infinite. (2) the length of the abstracted word may be too long. Both of them can make the automata learning too

So for practicality, we propose a three-layer abstraction, which consists of:

- value abstraction: each value in an input array is mapped into an integer via partitioning, which

- symbol abstraction: a block of multi-dimensional integer array is abstracted as a symbol, which

- word abstraction: the whole input array is encoded into a word, wherein value abstraction and

Value Abstraction. In order to reduce the size of alphabet, inspired by Omlin and Giles's work (Omlin and Giles, 1996), we first split the values of the input space into $n$ (equal) intervals, and map each interval into an integer, that is, the index of the corresponding intervals. Formally, let the input space be $\mathscr{I}$ and it be split into $n$ intervals $I_{0}, \ldots, I_{n-1}$. Then a value abstraction function $\alpha_{v}: \mathscr{I} \rightarrow\{0, \ldots, n-1\}$ is defined

$$
\forall d \in \mathscr{I} . \alpha_{v}(d)=i \text { such that } d \in I_{i}
$$

This value abstraction function maps a concrete value in the input to an abstract integer. Figure 4 shows an example of value abstraction applying on the array given in Figure 3 , where the input space $\mathscr{I}$ is $[0,1]$

While for concretization, an abstract integer is mapped to its corresponding interval, that is, the value concretization function $\gamma_{v}:\{0, \ldots, n-1\} \rightarrow 2^{\mathscr{I}}$ is defined:

Both $\alpha_{v}$ and $\beta_{v}$ can be extended on sets of elements in a natural way:

$$
f_{v}(S)=\bigcup_{d \in S} f_{v}(d)
$$

It is easy to get that $\alpha_{v}\left(\gamma_{v}(i)\right)=i$ for each integer $i$, and $d \in \gamma_{v}\left(\alpha_{v}(d)\right)$ for any given value $d$. Therefore, $\left(\alpha_{v}, \gamma_{v}\right)$ forms a Galois connection (Nielson et al., 1999). While in practice, especially when we query a word, we are unable to test all the values in the interval for each integer. For that, we randomly select at most $k_{v}$ (which can be dependent on the intervals) values to represent the corresponding interval. That is

$$
\gamma_{v}^{\text {prac }}(i)=\left\{d_{j} \mid d_{j} \in I_{i} \text { and } 0 \leq j<k_{v}\right\}
$$

Obviously, the larger $k_{v}$ is, the closer $\gamma_{v}^{\text {prac }}(i)$ is to $\gamma_{v}(i)$. So concerning Galois connections, the larger $k_{v}$,

But only Galois connections are not enough here. We also need to consider the safety of neural networks (Huang et al., 2017), that is, a vibration of values should not flap the outputs, since different values may be abstracted into an identity integer. In fact, the composition of the functions $\alpha_{v}$ and $\gamma_{v}$ can 
be viewed as a kind of manipulations (Huang et al., 2017). We say a $k$-value manipulation $v m^{k}$ with respect to $\alpha_{v}$ and $\gamma_{v}$ is a function such that for any input array in

$$
v m^{k}(\text { in })=i n \cdot\left[d_{i} \mapsto d_{i}^{\prime}\right]_{i \in\{1, \ldots, k\}}
$$

where $d_{i} \in$ in and $d_{i}^{\prime} \in \gamma_{v}\left(\alpha_{v}\left(d_{i}\right)\right)$. Intuitively, $k$-value manipulation replaces (at most) $k$ values of the input array by some values, which share the same intervals with the corresponding original values. Figure 5 shows an example of 4-value manipulation applying on the input array in Figure 4, where the input space $\mathscr{I}$ is $[0,1]$ and it is split into $I_{0}=[0,0.5)$ and $I_{1}=[0.5,1]$. And we say a network $\mathscr{N}$ is safe with respect to this value manipulation $v m^{k}$ if for every input array in

$$
\mathscr{N}\left(v m^{k}(\text { in })\right)=\mathscr{N}(\text { in })
$$

That is to say, performing this value manipulation should not result in a different classification. This requires that every interval $I_{i}$ should be as small as possible or the number $n$ of intervals should be as large as possible. However, the safety cannot easily be preserved in practice, unless the abstraction is an identity function or the network is robust enough. So instead, we use a weak notation called $\sigma$-safety: we say a network $\mathscr{N}$ is $\sigma$-safe with respect to $k$-value manipulation $v m^{k}$ under a given input set $D$ if

$$
\frac{\mid\left\{\text { in } \in D \mid \mathscr{N}\left(v m^{k}(\text { in })\right) \neq \mathscr{N}(\text { in })\right\} \mid}{|D|} \leq \sigma
$$
in blocks:

\begin{tabular}{|l|l|l|l|}
\hline 1 & 0 & 1 & 1 \\
\hline 0 & 1 & 1 & 1 \\
\hline 1 & 1 & 0 & 0 \\
\hline 1 & 0 & 0 & 1 \\
\hline
\end{tabular}

\begin{tabular}{|c|c|}
\hline 9 & 15 \\
\hline 14 & 1 \\
\hline
\end{tabular}

Figure 5. Example of $k$-Abstraction Manipulation Figure 6. Example of Base- $n$ Symbol Abstraction

Symbol Abstraction. After the value abstraction, each integer can be used as a symbol. But this could yield words that are too long to learn the model. So for scalability, we add a symbol abstraction, which abstracts input arrays into symbols by blocks. For simplicity, in this paper we consider 2-dimensional array with size $i R o w \times i C o l$. We say a slice of an input array starting from the index $(r i, c i)$ to the index $(r i+o$ Row $-1, c i+o C o l-1)$ is a block, and the size of the block is oRow $\times o$ Col.

A natural way to abstract blocks into symbols is to map the blocks into one dimension in row (or column) major order and then encode the one dimension into a base- $n$ number (or a string consisting of the integers in the one dimension). We denote this mapping as $\alpha_{s}^{B}$. Figure 6 gives an example of $\alpha_{s}^{B}$ that are performed on the array that are obtained by the value abstraction shown in Figure 4, where the number $n$ of intervals is 2 and the size of blocks is $2 \times 2$. Moreover, by decoding the base- $n$ number (or the string), it is easy to obtain the inverse mapping $\gamma_{s}^{B}$. It is clear $\left(\alpha_{s}^{B}, \gamma_{s}^{B}\right)$ forms a Galois connection. But a drawback of this solution is that the size of alphabet is $n^{o R o w \times o C o l}$, which could be too large in practice. For example, the size of alphabet of the example shown in Figure 6 is 16.

In this paper we use an alternative way to represent a block as its sum. In more detail, we define a symbol abstraction function $\alpha_{s}^{S}$ that maps integer blocks of size $o R o w \times o C o l$ into the sum of the integers

$$
\alpha_{s}^{S}(b)=\sum_{i \in b} i
$$

It is easy to compute the set of the possible sums of blocks, that is, $\{0, \ldots, o$ Row $\times o \mathrm{Col} \times(n-1)\}$. So the size of alphabet is $o$ Row $\times o \mathrm{Col} \times(n-1)+1$. Compared to the natural solution, the size of alphabet is quite smaller (from $n^{o R o w \times o C o l}$ reduced to $o$ Row $\times o C o l \times(n-1)+1$ ). Take the input array in Figure 6 for example. Under this sum abstraction, its abstraction is shown in Figure 7 and the size of alphabet is 5.

But it is pity that this mapping is not bijective. So in order to form Galois connections, similar to value abstraction, we define the inverse mapping from symbols (i.e. sums) to sets consisting of blocks of 


\begin{tabular}{|l|l|l|l|}
\hline 1 & 0 & 1 & 1 \\
\hline 0 & 1 & 1 & 1 \\
\hline 1 & 1 & 0 & 0 \\
\hline 1 & 0 & 0 & 1 \\
\hline
\end{tabular}$\quad \stackrel{\alpha_{s}^{S}}{\longrightarrow}$\begin{tabular}{|l|l|l|}
2 & 4 \\
\hline 3 & 1 \\
\hline
\end{tabular}

Figure 7. Example of Sum Symbol Abstraction

\begin{tabular}{|l|l|l|l|}
\hline 1 & 0 & 1 & 1 \\
\hline 0 & 1 & 1 & 1 \\
\hline 1 & 1 & 0 & 0 \\
\hline 1 & 0 & 0 & 1 \\
\hline
\end{tabular}$\stackrel{s m^{1}}{\longrightarrow}$\begin{tabular}{|l|l|l|l|l|}
\hline 0 & 0 & 1 & 1 \\
\hline 1 & 1 & 1 & 1 \\
\hline 1 & 1 & 0 & 0 \\
\hline 1 & 0 & 0 & 1 \\
\hline
\end{tabular}

Figure 8. Example of $k$-Shift Manipulation

size $o$ Row $\times o$ Col whose sum is exact the symbol:

$$
\gamma_{s}^{S}(\text { sum })=\left\{b_{j} \mid \sum_{i \in b_{j}} i=s u m\right\}
$$

Likewise, these two functions can be lifted to sets of elements in a natural way. It is easy to get that $\alpha_{s}\left(\gamma_{s}(\right.$ sum $\left.)\right)=$ sum for each symbol sum, and $b \in \gamma_{s}\left(\alpha_{s}(b)\right)$ for any given block $b$ of size oRow $\times o$ Col. Therefore, $\left(\alpha_{s}, \gamma_{s}\right)$ forms a Galois connection. While for practicality, similar to value abstraction $\gamma_{v}$, we use a weak sum concretization function:

$$
\gamma_{s}^{\text {Sprac }}(\text { sum })=\left\{b_{j} \mid \sum_{i \in b_{j}} i=\operatorname{sum} \text { and } 0 \leq j<k_{s}\right\}
$$

That is, we select at most $k_{s}$ (which can be dependent on the block size and $n$ ) blocks to represent the corresponding sum. Clearly, the larger $k_{s}$ is, the closer $\gamma_{s}^{\text {Sprac }}($ sum $)$ is to $\gamma_{s}^{S}($ sum $)$. So concerning Galois connections, the larger $k_{s}$, the better.

In addition, the composition of $\alpha_{s}^{S}$ and $\gamma_{s}^{S}$ forms a manipulation as well, and the network $\mathscr{N}$ should be safe with respect to this manipulation. Formally, we say a $k$-shift manipulation $s m^{k}$ with respect to $\alpha_{s}^{S}$ and $\gamma_{s}^{S}$ is a function such that for any input array in

$$
\operatorname{sm}^{k}(i n)=i n \cdot\left[b_{i} \mapsto b_{i}^{\prime}\right]_{i \in\{1, \ldots, k\}}
$$

where $b_{i}$ is a block of size $o$ Row $\times o$ Col belonging to in and $b_{i}^{\prime} \in \gamma_{s}^{S}\left(\alpha_{s}^{S}\left(b_{i}\right)\right)$. And a network $\mathscr{N}$ is safe with respect to $s m^{k}$ if for every input array in

$$
\mathscr{N}\left(\operatorname{sm}^{k}(\text { in })\right)=\mathscr{N}(\text { in })
$$

In a word, $k$-shift manipulation replaces (at most) $k$ blocks of the input array by some blocks, which share the same sums with the corresponding original blocks; and performing this $k$-shift manipulation should not result in a different classification. Similar to value manipulation, the safety cannot easily be preserved in practice. So we use a weak notation called $\sigma$-safety: a network $\mathscr{N}$ is $\sigma$-safe with respect to $s m^{k}$ under a given input set $D$ if

$$
\frac{\mid\left\{\text { in } \in D \mid \mathscr{N}\left(\operatorname{sm}^{k}(\text { in })\right) \neq \mathscr{N}(\text { in })\right\} \mid}{|D|} \leq \sigma
$$

where $|D| \geq 1$. Considering the safety, the distance between two blocks of the same sum or the size of block should be as small as possible. Figure 8 shows an example of 1-shift manipulation applying on the input array in Figure 7, where $n$ is 2 and the size of blocks is $2 \times 2$.

Finally, let us consider the alphabet size. As discussed above, the alphabet size for the abstraction function $\alpha_{s}^{S}\left(\alpha_{s}\right.$ resp.) is linear (exponential resp.) in the block size and the number of intervals $n$. So concerning the alphabet size, the smaller the block size and the interval number $n$, the better.

Word Abstraction. Finally, we split the input array into blocks, and map them into a sequence of symbols (i.e., a word) in row (or column) major order. Algorithm 1 shows the detail of the word abstraction function $\alpha_{w}$. The algorithm first invokes the value abstraction $\alpha_{v}$ to map the values in the input array into integers (Line 1). Then it slides over the integer array block by block (Lines $2-10$ ) and maps each block into a symbol by the symbol abstraction $\alpha_{s}^{S}$ (Line 6). Note that here we use a narrow slide on the input array, that is, the blocks to be abstracted are fully contained in the input array. One can use the wide slide with zero-padding as well. Just like the convolution operation of CNN, one can further 


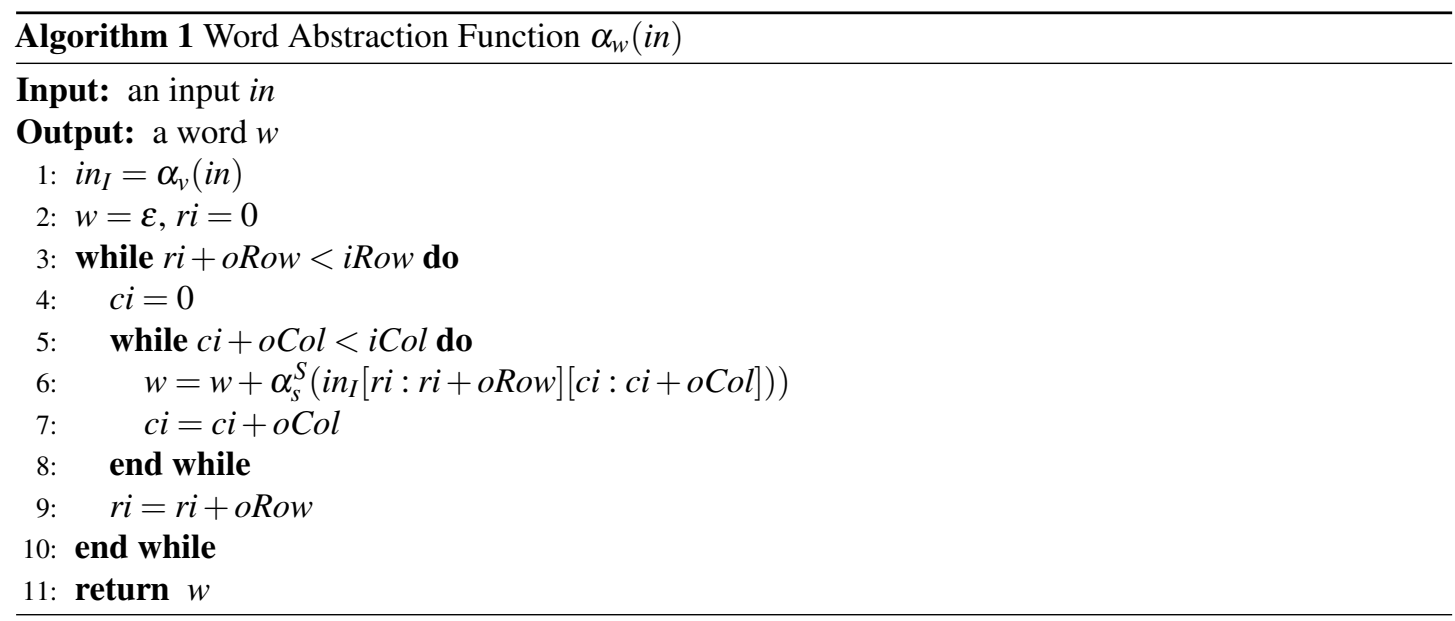

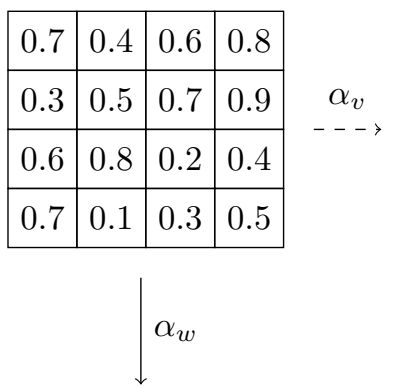

$2,4,3,1$ encoding practicality, we use set the stride sizes for each dimension. In addition, one can further encode the sequence of symbols into a final word in a more compact format, such as run-length encoding (RLE).

As mentioned above, the symbol abstraction aims to reduce the length of words. According to the word abstraction, we have that the larger the block size, the shorter the word.

Figure 9 shows our abstraction applying on the input array given in Figure 3, where the number $n$ of intervals is 2 and the size of blocks is $2 \times 2$. Compared to the simple abstraction shown in Figure 3 , our abstraction yields words with smaller size of alphabet and shorter length.

\begin{tabular}{|l|l|l|l|}
\hline 1 & 0 & 1 & 1 \\
\hline 0 & 1 & 1 & 1 \\
\hline 1 & 1 & 0 & 0 \\
\hline 1 & 0 & 0 & 1 \\
\hline
\end{tabular}

\begin{tabular}{|l|l|}
\hline 2 & 4 \\
\hline 3 & 1 \\
\hline
\end{tabular}

Figure 9. Example of Word Abstraction

The word concretization function $\gamma_{w}$, which is shown in Algorithm 2, does the opposite: it maps a sequence of symbols (i.e., a word) into a sequence of sets of blocks (Lines 5-7), and combines them into a set of arrays (Lines $8-10$ ). Note that we require the length of word to be concretized should conform to the size of input (Lines $2-4$ ). One can release this length condition by zero-padding or discarding the superfluous symbols. But this may break the Galois connections.

Theoretically, if $\left(\alpha_{v}, \gamma_{v}\right)$ and $\left(\alpha_{s}^{S}, \gamma_{s}^{S}\right)$ form Galois connections, then so does $\left(\alpha_{w}, \gamma_{w}\right)$. While for

$$
\gamma_{w}^{\text {prac }}=\gamma_{w}\left[\gamma_{v} \mapsto \gamma_{v}^{\text {prac }}, \gamma_{s}^{S} \mapsto \gamma_{s}^{\text {Sprac }}\right]
$$

instead, and the number of data in $\gamma_{w}^{\text {prac }}(w)$ depends on the word $w$ as well as $k_{v}$ and $k_{s}$. In particular, in our implementation we collect sets of inputs (including values and blocks) that are mapped into an identity word from existing data and then select inputs from the corresponding set.

Finally, considering safety, if $\left(\alpha_{v}, \gamma_{v}\right)$ and $\left(\alpha_{s}^{S}, \gamma_{s}^{S}\right)$ does not cause the flapping, then neither does $\left(\alpha_{w}, \gamma_{w}\right)$. But the safety of a network focus on the vibrations of local parts of inputs (Huang et al., 2017). To evaluate the whole inputs, we use another notation conflict, that is, inputs of different classifications should not abstracted into an identity word. Formally, we say a network $\mathscr{N}$ is non-conflict with respect to 


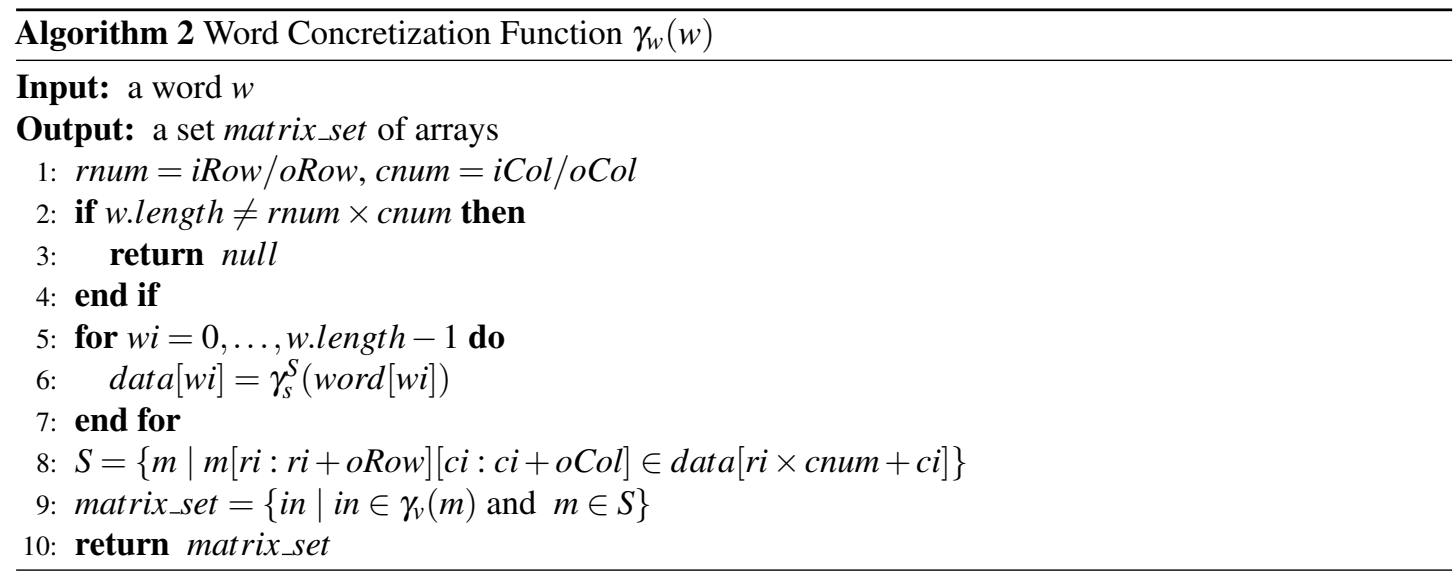

$\alpha_{w}$ and $\gamma_{w}$ if for every input array in and for every array $i n^{\prime} \in \gamma_{w}\left(\alpha_{w}(i n)\right)$

$$
\mathscr{N}\left(i^{\prime}\right)=\mathscr{N}(\text { in })
$$

In other words, the abstraction itself should not be over-approximated. We say a word $w$ is conflict, if there exist two inputs of different classifications that are abstracted into it. So to avoid over-approximation, the number of the conflict words caused by the abstraction should be as little as possible. Similar to the safety, the non-conflict cannot easily be preserved in practice. For that, we evaluate the conflicts words under a given dataset. In detail, we say a network $\mathscr{N}$ is $\sigma$-conflict with respect to $\alpha_{w}$ under a given set $D$ if

$$
\frac{\mid\left\{i n \mid \exists i n^{\prime} . \mathscr{N}(\text { in }) \neq \mathscr{N}\left(i n^{\prime}\right) \wedge \alpha_{w}(i n)=\alpha_{w}\left(i n^{\prime}\right)\right\} \mid}{|D|} \leq \sigma
$$

where $|D| \geq 1$ and in, $i^{\prime} \in D$.

To sum up, to obtain a suitable abstraction (e.g., scalable, safe and non-conflict), one needs to take into account the number $n$ of intervals, the block size oRow and $o \mathrm{Col}$, and the other factors.

\subsection{Active Learning}

In this section, we present how to instantiate the active learning framework on neural networks, in particular the membership and equivalence queries.

Membership Query. Membership queries can be answered by the neural networks via the word concretization function. In our abstraction, we map a word into a set of data. As mentioned above, the abstraction may flap the results or yield some conflict words, that is, the classifications of different data in the set of an identity word may not be the same. To address this, we count the numbers of different classifications of the data in the set and take the classification which gets the most votes as the result for the word.

Given a network $\mathscr{N}$ and a word concretization function $\gamma_{w}$, we say a word $w$ is positive if

$$
\mid\left\{\text { in } \in \gamma_{w}(w) \mid \mathscr{N}(\text { in })=\text { true }\right\}|\geq|\left\{\text { in } \in \gamma_{w}(w) \mid \mathscr{N}(\text { in })=\text { false }\right\} \mid
$$

and otherwise negative. Intuitively, a word is positive (negative resp.) if there are more positive (negative resp.) input arrays that are abstracted into it than the negative (positive resp.) ones.

Algorithm 3 gives the procedure of membership query checking, where $\mathscr{N}$ denote the neural network under learning. Firstly, the algorithm concretises the word $w$ that is being queried into a set matrix_set of possible data, using the word concretization function $\gamma_{w}$ (Line 1). If the set matrix_set is null, that is, the length of word $w$ does not conform to the size of input data, then the algorithm returns false immediately. Otherwise, the algorithm feeds each data into the neural network $\mathscr{N}$ under learning and counts the numbers of different classifications (Lines 5-12). Finally, it returns the classification that gets the most votes (Line 13).

Equivalence Query. As there is no finite interpretation for neural networks (Weiss et al., 2018), equivalence queries are more challenging than membership queries. To address this, similar to Weiss et al.'s work (Weiss et al., 2018), we use an abstract representation of the neural network under learning. 


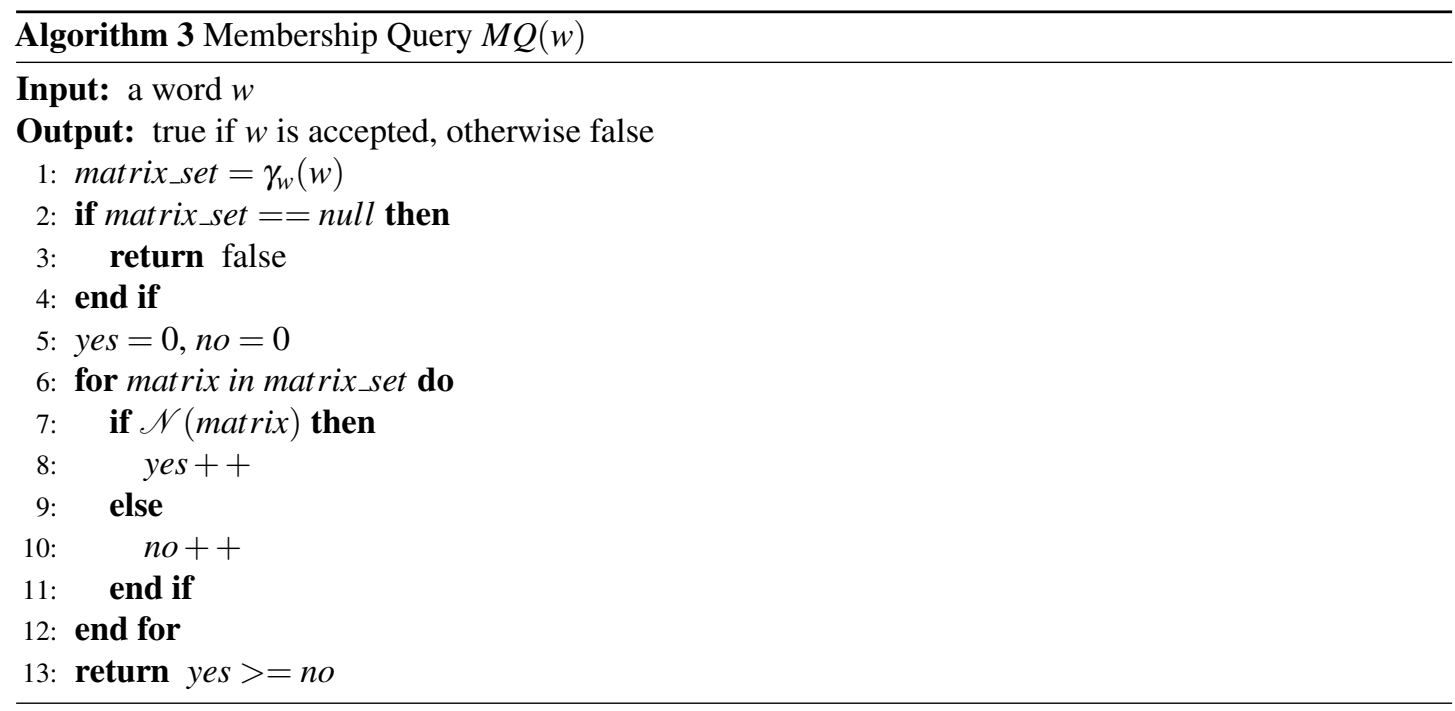

But different from Weiss et al.'s work (Weiss et al., 2018), we start with the automaton that is learned passively via the regular positive and negative inference (RPNI) algorithm (Oncina and García, 1992) from some test queries, which are selected from the training dataset. Then we perform the equivalence query against this abstract model. As discussed in (Weiss et al., 2018), when a counterexample is found, it may be not that the hypothesis is incorrect, but rather that the abstract model is not precise enough (i.e., different behaviors from the neural network under learning) and needs to be refined.

The procedure ${ }^{3}$ of equivalence query checking is given in Algorithm 4. Firstly, the algorithm tries to find a word that can separate the hypothesis $\mathscr{H}$ and the abstract model $\mathscr{M}$ (Line 3 ). If such a word does not exist, then it returns null (Lines 4-6), which means the equivalence query is yes. Assume a word $w$ is found. Then it checks whether this word is a true counterexample, that is, the classifications of the abstract model and the neural network under learning are the same (Line 7). If it is in that case, then it returns this word as a counterexample to the learner (Line 8). Otherwise, it refines the abstract model with this word (Line 10): it adds the counterexample into the positive set or the negative set dependent on its true classification, and relearns a new automata via RPNI. After that, the algorithm continues on the equivalence query against this refined model.

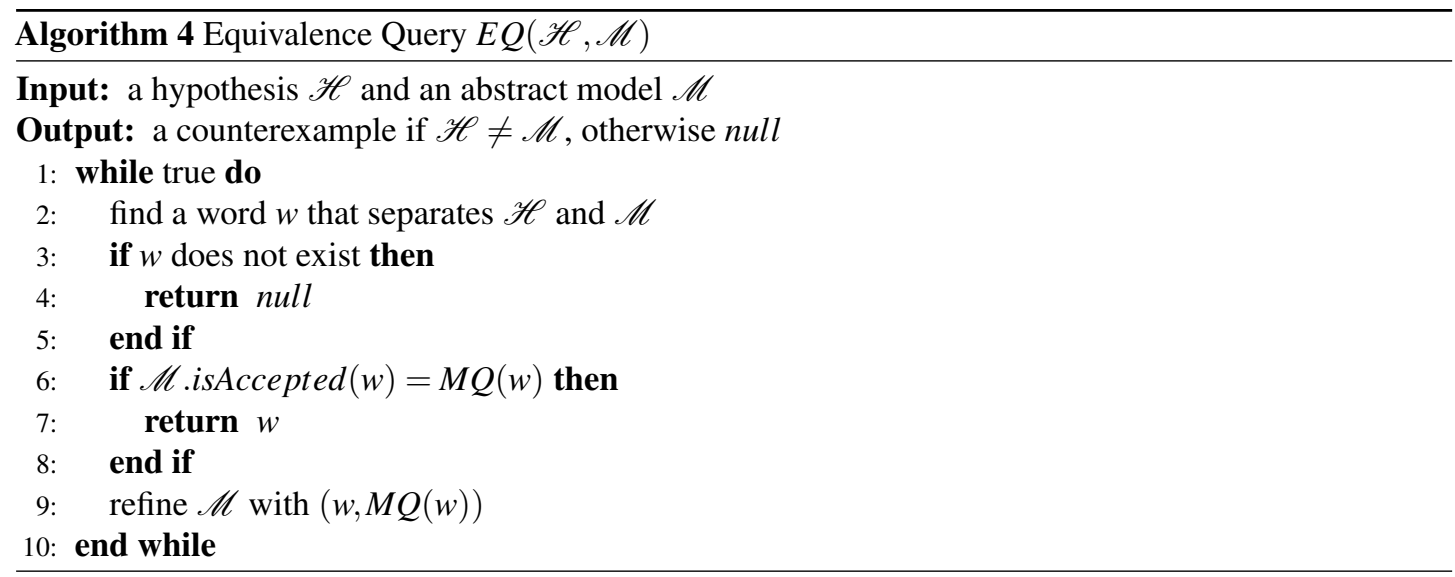

\section{EXPERIMENTS}

We have implemented our approach in a prototype in Java, wherein we use the library LearnLib (Howar et al., 2012) to implement the MAT learning framework and the RPNI algorithm. Moreover, to find

\footnotetext{
${ }^{3}$ In our implementation, we set a bound for the refining time for efficiency, which may yield an incompatible acceptance
} exception. 
Table 1. Flapped results on different $k$-value manipulations

\begin{tabular}{|c|c|c|c||c|c|c|c|}
\hline$n$ & $k$ & Flaps & Ratio & $n$ & $k$ & Flaps & Ratio \\
\hline \hline 2 & 1 & 3 & $0.005 \%$ & 2 & 10 & 6 & $0.010 \%$ \\
\hline 2 & 100 & 32 & $0.053 \%$ & 2 & 500 & 276 & $0.461 \%$ \\
\hline 3 & 1 & 0 & $0.000 \%$ & 3 & 10 & 5 & $0.008 \%$ \\
\hline 3 & 100 & 16 & $0.027 \%$ & 3 & 500 & 43 & $0.072 \%$ \\
\hline 5 & 1 & 0 & $0.000 \%$ & 5 & 10 & 0 & $0.000 \%$ \\
\hline 5 & 100 & 10 & $0.017 \%$ & 5 & 500 & 16 & $0.027 \%$ \\
\hline 10 & 1 & 1 & $0.002 \%$ & 10 & 10 & 0 & $0.000 \%$ \\
\hline 10 & 100 & 5 & $0.008 \%$ & 10 & 500 & 6 & $0.015 \%$ \\
\hline
\end{tabular}

\footnotetext{
${ }^{4}$ The other equivalence approximation strategies provided in LearnLib can be used as well.

${ }^{5}$ https: //github. com/deeplearning4j/dl 4 j-examples.

${ }^{6}$ http://yann. lecun. com/exdb/mnist/.
} provided in LearnLib and the MNIST classifier itself. 32GB memory.

\subsection{MNIST Classifier} official site ${ }^{6}$, wherein each input is 2-dimensional integer matrix with size $28 \times 28$.

\subsection{Abstraction Experiments} abstractions with different interval numbers and block sizes. different block sizes. the number of flapped input to the total number of selected inputs.

the true counterexamples faster, we use the Wp-method test (Fujiwara et al., 1991) ${ }^{4}$ in the equivalence query between the hypothesis and the abstract models. To evaluate our approach, we conduct a series of experiments on a classifier for the MNIST dataset, a large database of handwritten digits that is commonly used for training various image processing systems. Firstly, we conduct experiments to see the measures of the MNIST classifier, namely, $\sigma$-safety, $\sigma$-conflict, the size of alphabet and the length of words, under the abstractions with different interval numbers and block sizes. Secondly, we present the experiments to learn DFAs from the MNIST classifier under different selected abstractions. Thirdly, we also conduct experiments to compare the resulted DFAs against the DFAs learned via the passive learning algorithms

The experiments were conducted on a workstation with Intel Processor i7-7820HQ (2.90GHz) and

The MNIST classifier under learning is a binary classification version of MnistClassifier from the tutorial examples of DeepLearning $4 \mathrm{~J}^{5}$, which recognises the number 1. It is built on a convolution neural network, which consists of 6 layers, namely, a convolution layer, a pooling layer, another convolution layer, another pooling layer, a dense layer and an output layer. The training dataset and the testing dataset are from the

As discussed in Section 3.1, the interval number $n$ and the block size affect the definition of the abstraction, especially the safety and the conflict of the neural network under learning, the size of alphabet and the length of words. For that, we present in this section some experiments to see these measures of the

Safety. The first measure to test is the safety. For that, we present some experiments to test the flapping of the MNIST classifier on some selected inputs from the training set (i) via performing $k$-value manipulations $v m^{k}$ with different interval numbers and (ii) via performing $k$-shift manipulations $s m^{k}$ with

First, in the experiments about $k$-value manipulation $v m^{k}$, for a given interval number $n$, we randomly select $k$ values from a selected input, and replace each selected value by a random value which shares the same interval with the corresponding selected value. Then we fed the resulted data into the MNIST classifier and see whether the classifications are flapped. We select 59838 inputs in total from the training set, which are classified correctly by the MNIST classifier. Table 1 shows the results, where Flaps denotes the number of inputs whose results are flapped by the manipulation, and Ratio denotes the percentage of

From the results we can see that, the number of flapped inputs increases as the number $k$ of selected values increases, since the larger the number $k$, the larger the vibration for the inputs. In contrast, as the number of intervals increase, the number of flapped inputs decreases, which indicates that the larger the interval number, the better. This conforms to the discussion in Section 3.1. Moreover, the results also 
Table 2. Flapped results on $k$-shift manipulations

\begin{tabular}{|c|c|c|c|c||c|c|c|c|c|}
\hline oRow & $o$ Col & $k$ & Flaps & Ratio & oRow & $o$ Col & $k$ & Flaps & Ratio \\
\hline \hline 1 & 28 & 1 & 0 & $0.000 \%$ & 28 & 1 & 1 & 4 & $0.007 \%$ \\
\hline 1 & 28 & 4 & 33 & $0.055 \%$ & 28 & 1 & 4 & 376 & $0.628 \%$ \\
\hline 1 & 28 & 28 & 5136 & $8.583 \%$ & 28 & 1 & 28 & 6608 & $11.043 \%$ \\
\hline 2 & 28 & 1 & 10 & $0.017 \%$ & 28 & 2 & 1 & 105 & $0.175 \%$ \\
\hline 2 & 28 & 2 & 109 & $0.182 \%$ & 28 & 2 & 2 & 879 & $1.469 \%$ \\
\hline 2 & 28 & 14 & 5768 & $9.639 \%$ & 28 & 2 & 14 & 6615 & $11.055 \%$ \\
\hline 4 & 28 & 1 & 382 & $0.638 \%$ & 28 & 4 & 1 & 1018 & $1.701 \%$ \\
\hline 4 & 28 & 7 & 6284 & $10.502 \%$ & 28 & 4 & 7 & 6617 & $11.058 \%$ \\
\hline 7 & 28 & 1 & 1273 & $2.127 \%$ & 28 & 7 & 1 & 2077 & $3.471 \%$ \\
\hline 7 & 28 & 4 & 6684 & $11.170 \%$ & 28 & 7 & 4 & 6620 & $11.063 \%$ \\
\hline 14 & 28 & 1 & 3050 & $5.097 \%$ & 28 & 14 & 1 & 3766 & $6.294 \%$ \\
\hline 14 & 28 & 2 & 6436 & $10.756 \%$ & 28 & 14 & 2 & 6431 & $10.747 \%$ \\
\hline 28 & 28 & 1 & 6425 & $10.737 \%$ & - & - & - & - & - \\
\hline
\end{tabular}

show that the MNIST classifier is about $0.053 \%$-safety, with respect to the 100 -value manipulation $\mathrm{vm}^{100}$ with the interval number 2 . And the 100-value manipulation means $12.76 \%(100 / 784)$ of an input has been modified, such that we believe 100 is enough for local vibration. Therefore, we suggest to set the interval number $n$ as 2 .

Next, in the experiments about $k$-shift manipulation $s m^{k}$, for a given block size $o$ Row $\times o C o l$, we randomly select $k$ blocks from a selected input, and rearrange the values in each selected block. Then we feed the resulted data into the MNIST classifier and see whether the classifications are flapped. Similarly, we select the 59838 inputs that are classified correctly by the MNIST classifier from the training set. For simplicity and scalability, we consider the block sizes whose row sizes or column sizes are 28 . The results are given in Table 2, where the notations are the same as the ones in Table 1.

First, the results show that, as the size of block increases, the number of flapped inputs increases, which conforms to the discussion in Section 3.1. The results also show that the number of flapped inputs increases as the number $k$ of selected blocks increases. This is because that, the larger the number $k$, the larger the vibration for the inputs. Moreover, we found that the MNIST classifier is more safe under $k$-shift manipulation built on rows than the one on columns. The reason may be that the digit number of 1 is more regular in row order than in column order. Finally, assume the size allowed for local vibration is about 100. All the $\sigma$-safeties of the MNIST classifier with respect to the $k$-shift manipulation with the block size $1 \times 28,2 \times 28,4 \times 28,28 \times 1,28 \times 2$ or $28 \times 4$ are smaller than $1.8 \%$. In particular, the MNIST classifier is about $0.055 \%$-safety, with respect to the 4-shift manipulation $v m^{4}$ with the block size $1 \times 28$.

Conflict. The second measure to test is the non-conflict, which indicates whether the abstraction with the given block size is over-approximated. In other words, we would like to conduct experiments to test how many conflict words that are generated by the abstractions with different block sizes under the training set. For that, we perform the abstractions with different block sizes on some selected inputs from the training set, and do a statistic analysis on the abstracted words with respect to their classifications, where we take the interval number $n$ as the suggested one 2 . The test inputs that are selected from the training set is 59840 in total, with 6700 positive inputs and 53140 negative ones.

The statistic results are given in Table 3, where TW denotes the total number of words, PW (NW resp.) denotes the number of positive (negative resp.) words, CPW ( CNW resp.) denotes the number of positive (negative resp.) words that have both positive and negative inputs and CPD (CND resp.) denotes the number of positive (negative resp.) inputs that are abstracted into a negative (positive resp.) word.

The results show that as the block size increases, the number of abstracted words decreases, which conforms to the discussion in Section 3.1. Thus it could be easier to extract the automaton for a larger block size. For example, when taking the whole input as a symbol, there are 239 words in total. But both the number of conflict words and the number of conflict data increase as the block size increases, which indicates that an abstraction with a larger block size is prone to be an over-approximation. In particular, when taking the whole input as a symbol, $56.194 \%$ of the positive inputs are abstracted into negative words and $64.865 \%$ of the positive words are conflict. Moreover, from the results we can also see that all 
Table 3. Conflict results on different abstractions

\begin{tabular}{|c|c|c|c|c|c|c|c|c|}
\hline oRow & $o \mathrm{Col}$ & TW & PW & NW & CPW & CNW & CPD & CND \\
\hline \hline 1 & 28 & 59745 & 6605 & 53140 & 0 & 0 & 0 & 0 \\
\hline 2 & 28 & 59640 & 6500 & 53140 & 0 & 0 & 0 & 0 \\
\hline 4 & 28 & 58817 & 5755 & 53062 & 15 & 0 & 0 & 15 \\
\hline 7 & 28 & 53912 & 4501 & 49411 & 246 & 11 & 11 & 248 \\
\hline 14 & 28 & 5444 & 248 & 5196 & 163 & 544 & 2577 & 1367 \\
\hline 28 & 1 & 59708 & 6569 & 53139 & 1 & 0 & 0 & 1 \\
\hline 28 & 2 & 59321 & 6195 & 53126 & 8 & 0 & 0 & 8 \\
\hline 28 & 4 & 55944 & 4512 & 51432 & 421 & 80 & 81 & 431 \\
\hline 28 & 7 & 36535 & 1414 & 35121 & 490 & 744 & 2276 & 1333 \\
\hline 28 & 14 & 5229 & 734 & 4495 & 327 & 871 & 3196 & 1195 \\
\hline 28 & 28 & 239 & 37 & 202 & 24 & 79 & 3765 & 1204 \\
\hline
\end{tabular}

Table 4. Word complexities on different abstractions

\begin{tabular}{|c|c|c|c|c|c||c|c|c|c|c|c|}
\hline$n$ & oRow & $o$ Col & dSize & Size & Length & $n$ & oRow & $o$ Col & dSize & Size & Length \\
\hline \hline 2 & 1 & 28 & 21 & 29 & 28 & 2 & 28 & 1 & 21 & 29 & 28 \\
\hline 2 & 2 & 28 & 41 & 57 & 14 & 2 & 28 & 2 & 41 & 57 & 14 \\
\hline 2 & 4 & 28 & 48 & 113 & 7 & 2 & 28 & 4 & 81 & 113 & 7 \\
\hline 2 & 7 & 28 & 126 & 197 & 4 & 2 & 28 & 7 & 124 & 197 & 4 \\
\hline 2 & 14 & 28 & 143 & 393 & 2 & 2 & 28 & 14 & 146 & 393 & 2 \\
\hline 2 & 28 & 28 & 244 & 785 & 1 & - & - & - & - & - & - \\
\hline
\end{tabular}

the $\sigma$-conflicts for the MNIST classifier with respect to the abstractions with the block size $1 \times 28,2 \times 28$, $28 \times 1$ or $28 \times 2$ are smaller than $0.015 \%(8 / 59840)$. And the abstractions with the block size $2 \times 28$ and $1 \times 28$ perform best, yielding none conflict data nor words.

Word Complexities. Finally, we also conduct experiments to see the size of alphabets and the length of words, which are dubbed as word complexities. Table 4 shows the word complexities under different abstractions with different block sizes, where Size denotes the size of alphabet, dSize denotes the number of symbols occurring in the selected inputs, and Length denotes the length of words.

The results show that the larger the block size, the larger the alphabet size and the shorter the word length, which conforms to the discussion in Section 3.1. Moreover, we found that the products of the alphabet size and the word length are almost the same. So for scalability, any block size seems fine. But if considering the practical alphabet (i.e., symbols occurring in the inputs), the larger block size could be better.

To sum up, based on the experiments above, we suggest to use for the MNIST classifier the abstractions with the interval number $n=2$ and the block size $1 \times 28,2 \times 28,28 \times 1$ or $28 \times 2$.

\subsection{Automata Learning}

In this section, we present the experiments to learn DFAs from the MNIST classifier under the suggested abstractions.

To quantitatively validate the models, we use the following performance measures. Accuracy is the most intuitive performance measure and it is simply a ratio of correctly predicted observation to the total observations. Precision is the ratio of correctly predicted positive observations to the total predicted positive observations, and Recall is the ratio of correctly predicted positive observations to all observations in actual class. F1 score is the weighted average of Precision and Recall, that is, $(2 \cdot$ Precision $\cdot$ Recall $) /($ Precision + Recall $)$. Moreover, there are two kinds of observations in our experiments, namely, the input arrays and the abstracted words (i.e., the abstractions of the input arrays). So we compute the measures above with respect to both kinds of observations. Intuitively, the higher the measures above, the better the model.

Automata Performance. We first conduct experiments to learn DFAs from the MNIST classifier under different suggested abstractions. Then we perform experiments to evaluate the learned DFAs on 
Table 5. Automata Performance under different abstractions

\begin{tabular}{|c|c|c|c|c|c|c|c|c|c|}
\hline oRow & $o$ Col & wPre & wRec & wAcc & wF1 & dPre & dRec & dAcc & dF1 \\
\hline \hline 1 & 28 & $55.041 \%$ & $70.782 \%$ & $90.176 \%$ & $61.927 \%$ & $55.288 \%$ & $70.988 \%$ & $90.184 \%$ & $62.162 \%$ \\
\hline 2 & 28 & $54.830 \%$ & $70.321 \%$ & $90.142 \%$ & $61.617 \%$ & $55.203 \%$ & $70.635 \%$ & $90.154 \%$ & $61.973 \%$ \\
\hline 28 & 1 & $44.382 \%$ & $70.035 \%$ & $86.691 \%$ & $54.333 \%$ & $44.569 \%$ & $70.194 \%$ & $86.699 \%$ & $54.521 \%$ \\
\hline 28 & 2 & $42.904 \%$ & $69.686 \%$ & $86.232 \%$ & $53.110 \%$ & $43.466 \%$ & $70.106 \%$ & $86.248 \%$ & $53.662 \%$ \\
\hline 28 & 28 & $100.000 \%$ & $66.667 \%$ & $94.064 \%$ & $80.000 \%$ & $74.472 \%$ & $40.388 \%$ & $91.657 \%$ & $52.373 \%$ \\
\hline
\end{tabular}

Table 6. Learning Complexities under different abstractions

\begin{tabular}{|c|c|c|c|c|c|c|c|}
\hline oRow & $o$ Col & aTime & xTime & iTime & aState & xState & iState \\
\hline \hline 1 & 28 & 29150.5 & 47761.8 & 16393.2 & 579 & 949 & 251 \\
\hline 2 & 28 & 8145.3 & 15833.9 & 2838.4 & 299.3 & 495 & 139 \\
\hline 28 & 1 & 16164.0 & 65525.8 & 75.7 & 801.1 & 2213 & 239 \\
\hline 28 & 2 & 6492.4 & 12650.5 & 214.6 & 309.3 & 499 & 97 \\
\hline 28 & 28 & 5.9 & 11.8 & 0.1 & 2 & 2 & 2 \\
\hline
\end{tabular}

the testing dataset. The performance results of the learned DFAs are given in Table 5, where the interval number $n$ is 2, the columns wAcc, wPre, wRec and $\mathbf{w F 1}$ respectively denote the Accuracy, Precision, Recall and F1 score that are computed with respect to words, and the columns dAcc, dPre, dRec and dF1 respectively denote the Accuracy, Precision, Recall and F1 score that are computed with respect to input data.

The results show that all the learned DFAs perform well on the testing dataset, with the $F 1$ score more than $50 \%^{7}$. In particular, the DFAs learned under the abstraction with block size $1 \times 28$ performs best. The results also show that DFA learned via the abstraction with a smaller block size can obtain a higher F1 score. In detail, the $F 1$ score of DFA learned via the abstraction with block size $1 \times 28(28 \times 1$ resp.) is higher than the one with block size $2 \times 28$ or $28 \times 2(28 \times 2$ resp.). This is because that a smaller block size can generate a more preciser abstraction, which conforms to the discussion in Section 3.1. Moreover, from the results, we can see that the DFAs learned under the abstractions in rows perform better than the ones under the abstractions in columns in terms of all performance measures with respect to both words and data. For example, the $F 1$ score of DFA learned via the abstraction with block size $1 \times 28$ is higher than the one with block size $28 \times 1$. The reason may be that the digit number of 1 is more regular in row order than in column order. In addition, we also perform the abstraction mapping a whole input as a symbol as does in Weiss et al.'s work (Weiss et al., 2018). Due to this abstraction is over-approximated, the extracted DFA gets the worst performance in the input data layer, although it has a better performance than the other models on the word layer, especially the Precision.

Learning Complexities. During the experiments, we also count the learning times in seconds needed by the resulted DFAs and the sizes of the resulted DFAs. The results are given in Table 6, where aTime, xTime and iTime respectively denote the average time, the maximum time and the minimum time needs by the resulted DFAs, and aState, $\mathbf{x S t a t e}$ and iState respectively denote the average number, the maximum number and the minimum number of states of the resulted DFAs.

From the results, we can see that learning the DFA via a smaller block size needs more time. As discussed before, an input array can be abstracted into a longer word under the abstraction with a smaller block size, which thus requires more time to proceed. Concerning the size of learned DFA, learning via a smaller block size can yield a larger DFA. For example, the number of the states of the DFA learned under the abstraction with block size $28 \times 1$ is the largest one among the results. Similar to the learning time, the reason is that an abstraction with a smaller block size yields longer words, which could enlarge the learned DFA. In addition, both the learning times and the sizes of the learned DFAs under the abstractions in rows are larger than the ones under the abstractions in columns. The reason may be that more blocks are abstracted into 0 under the abstractions in columns than the abstractions in rows.

Learned Automata. Finally, we convert the learned DFAs into the format used in JFLAP (Rodger

\footnotetext{
${ }^{7}$ Assume a random binary classifier predicts half of the digits as 1 with the accuracy $50 \%$. As the ratio of digit 1 in the dataset is about $1 / 10$, the $\mathrm{F} 1$ score is about $16.7 \%$.
} 
and Finley, 2006), which enables us to view the DFAs and convert DFA into regular expressions step by step. For simplicity, we consider a DFA learned via the abstraction with block size $14 \times 28$, which is given in Figure 10. From this figure, we can see that $(i)$ the learned DFA has a very clear structure: a starting node, an intermediate layer with several nodes, an accepting node and a trap node; and (ii) the learned DFA accepts words with length 2. To understand it further, we convert this DFA into a regular expression, which is a union of several alternatives. Among these alternatives, some of them are easy to understand and explain. For example, the expressions $(31+29)(31+32+30+29+33), 25(23+31+$ $26+27+28+29+24+25+30)$ and $26(30+31+32+26+27+28+29+25+33+24+34)$ state that the upper part and the low part share similar sums.

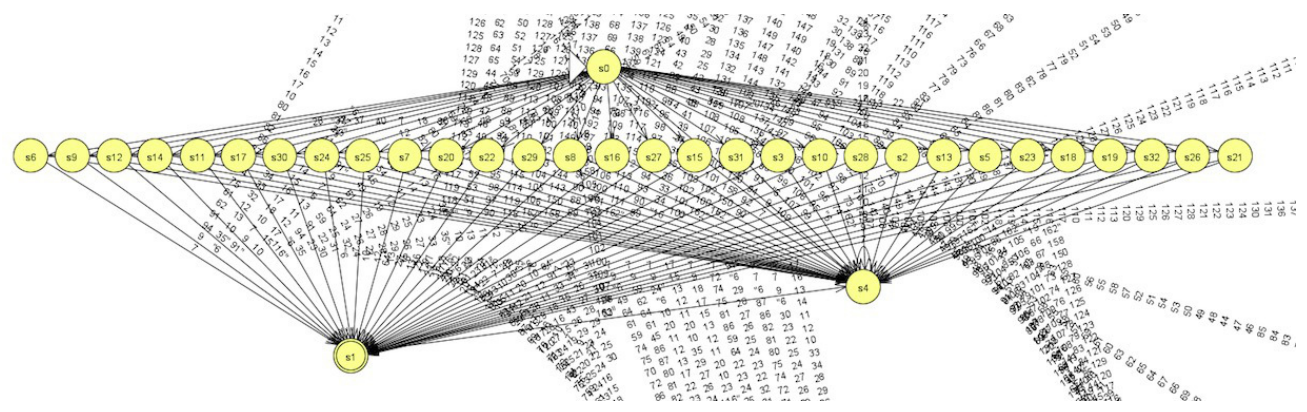

Figure 10. DFA Learned via Abstraction with $14 \times 28$

Let us see a DFA learned via the abstraction with block size $7 \times 28$, which is shown in Figure 11 . Compared with the one in Figure 10, this DFA has a more complex structure. But we still can identity some hierarchical structures in it. It is pity that we are not able to convert this DFA into a regular expression via JFLAP due to a runtime error.

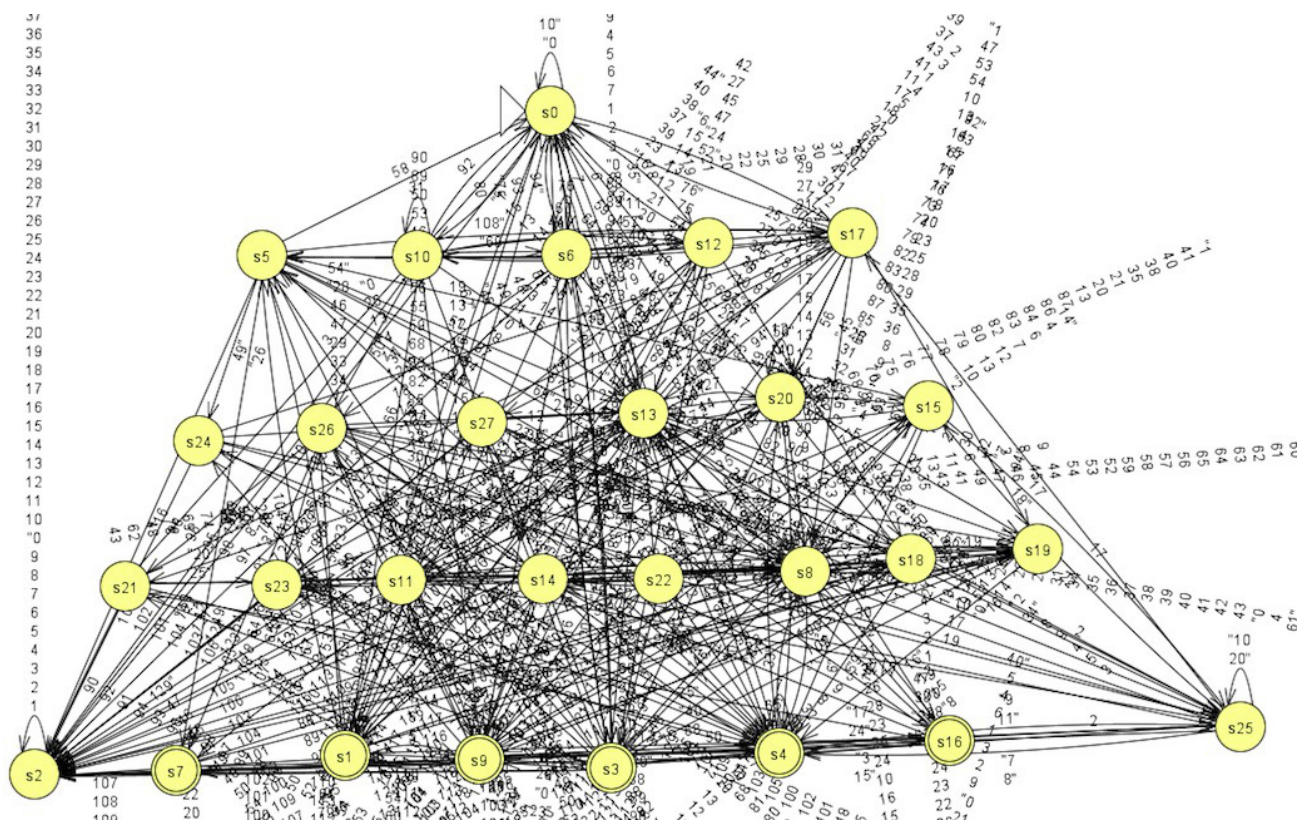

Figure 11. DFA Learned via Abstraction with $7 \times 28$

In addition, we also present a DFA learned via the abstraction with block size $1 \times 28$, which has 949 states and is given in Figure 12. It is a little complex to understand, so we can only identity a rough hierarchical structure. We believe that one can understand this DFA more if he gets a closer look on it. In addition, the learned DFAs can help to generate test cases to test the networks, which are left as a future work. 


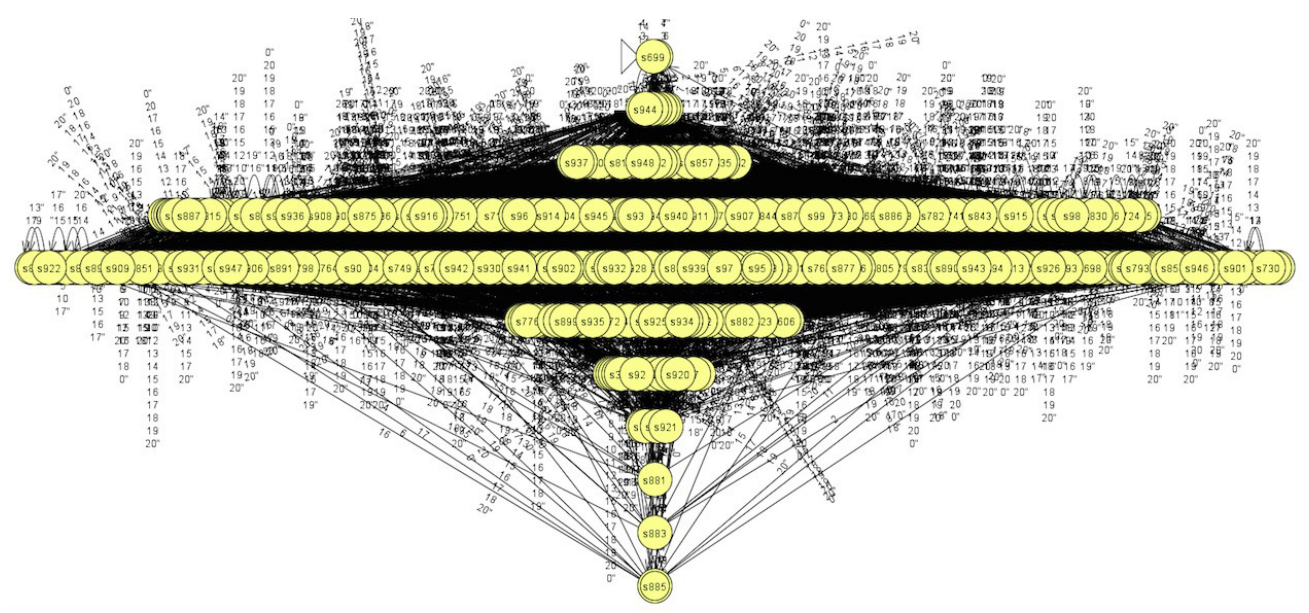

Figure 12. DFA Learned via Abstraction with $1 \times 28$

Table 7. Comparison against the passive DFAs and the MNIST classifier

\begin{tabular}{|c|c|c|c|c|c|c|c|c|}
\hline Model & wPre & wRec & wAcc & wF1 & dPre & dRec & dAcc & dF1 \\
\hline \hline Ours & $55.041 \%$ & $70.782 \%$ & $90.176 \%$ & $61.927 \%$ & $55.288 \%$ & $70.988 \%$ & $90.184 \%$ & $62.162 \%$ \\
\hline RPNI & $46.170 \%$ & $59.947 \%$ & $87.590 \%$ & $52.164 \%$ & $46.463 \%$ & $60.229 \%$ & $87.600 \%$ & $52.458 \%$ \\
\hline RPNI-MDL & $15.599 \%$ & $99.911 \%$ & $38.974 \%$ & $26.985 \%$ & $15.693 \%$ & $99.912 \%$ & $39.022 \%$ & $27.125 \%$ \\
\hline RPNI-EDSM & $19.407 \%$ & $98.313 \%$ & $53.729 \%$ & $32.416 \%$ & $19.520 \%$ & $98.325 \%$ & $53.766 \%$ & $32.574 \%$ \\
\hline CNN & - & - & - & - & $99.69 \%$ & $99.40 \%$ & $99.06 \%$ & $98.63 \%$ \\
\hline
\end{tabular}

\subsection{Comparison}

To further evaluate the resulted DFA, we compare it against the DFAs learned via the passive learning algorithms provided in LearnLib, namely, the RPNI algorithm, the RPNI-EDSM algorithm and the RPNI-MDL algorithm, and the MNIST classifier itself. In these experiments, we perform our abstraction on the training data and then learn a DFA via each passive learning algorithms provided in LearnLib, wherein the abstraction we used is the one with interval number 2 and block size $1 \times 28$, all the arrays are selected for the RPNI algorithm, only the positive arrays are selected for the RPNI-MDL algorithm (since it does not support the negative examples), and all the positive arrays and only one ninth of the negative ones are selected for the RPNI-EDSM algorithm (to avoid memory overflow and time-consuming). Next, we evaluate all the models with the testing dataset. The results are given in Table 7, where the notations are the same as the ones of Table 5.

Compared to the RPNI one, our DFA performs better in all the performance measures, since the abstract model we use is the DFA learned via the RPNI algorithm from some inputs in the training dataset and is refined with respect to the classifier continually during learning. While compared to the RPNI-MDL and RPNI-EDSM ones, our DFA has a better Accuracy, Precision and F1 score, but a worse Recall. This is because that these two DFAs take all the positive inputs in the training set into account such that it can recognise more positive inputs in the testing dataset, while only part of positive inputs are selected for our abstracted model. The results also show that our DFA is still worse than the classifier. There are several reasons for this. The first one is that we have set some bounds (e.g., the refining time for the abstract representation) in our implementation for the learning procedure for efficiency and to avoid memory overflow. The second one is that the Wp-method test used in our experiments may miss some true counterexamples. The third one is that the abstraction may be over-approximated to yield too many conflict words. Nevertheless, our approach still needs to be improved.

\section{LIMITATIONS}

Although our approach works for the MNIST classifier, there are still some limitations. Firstly, to figure out a suitable abstraction for the neural network under learning is not an easy task. As shown in (Biggio et al., 2013; Szegedy et al., 2013; Huang et al., 2017), several deep neural networks, including highly 
trained and smooth networks optimised for vision tasks, are unstable with respect to so called adversarial perturbations. Hence, some neural networks may be too sensitive to the abstraction manipulation to find a reasonable interval number. Even if a reasonable interval number were found, one need to make a compromise between the abstraction and the scalability to find a block size. Moreover, whether a turing machine can simulate a natural neural network is an open question (Zenil and Quiroz, 2006). So in some sense, we cannot define an abstraction without the conflict or the flapping.

Secondly, the scalability is another problem. Generally, the size of inputs of neural networks is in thousands. For such a neural network, either the alphabet may be too large (if a large block size is taken) or the word may be too long (if a small block size is taken) for us to extract the automaton. Taking the MNIST classifier for example, it may last several hours for some abstractions (see the abstraction with block size $1 \times 28$ in Table 6 ) to extract the automaton, even several days. There are two possible reasons for this issue in our implementation: (i) we use the implementation of RPNI algorithm from LearnLib, which does not support incremental learning proposed in (Dupont, 1996) and can be improved with it; and (ii) there are too many queries for the non-accepting words with invalid lengths.

Thirdly, our approach is dependent on the dataset. In Section 4, we selected the interval number and the block size via an analysis on the training dataset. Different datasets may derive different abstractions. To make things worse, it may be the case that an abstraction is suitable for the training dataset, but unsuited for some other testing dataset. Moreover, our abstract model is built from some existing testing data. Different data yields different abstract models, which could affect the results, such as the learning time and the learned DFA.

Fourthly, the implementation of equivalence query is a practical problem requiring attention. One may think that a precise equivalence check can be performed in polynomial time on the hypothesis automaton and the abstract automaton. However, the precise equivalence check could return too many false counterexamples such that it takes too much time for the learning. This is because the precise equivalence check is prone to generate a short and false counterexample that is invalid with respect to the abstraction. Indeed, we have tried this precise equivalence check, but we only succeed on the abstraction of $28 \times 28$ in one hour. So considering the efficiency, we use the Wp-method test in the equivalence check, which enables us to find the counterexamples whose lengths are in a given range.

Fifthly, although our approach is black-box, the structures of neural networks may affect the performances of the networks themselves, so as the performances of the learned DFAs. We have performed our approach with the abstraction $2 \times 28$ on the MNIST classifiers whose hidden layer numbers range in $\{1,2,5,10\}$, which are binary classification versions from the tutorial examples of DeepLearning $4 \mathrm{~J}$. The accuracies of all the classifiers are above 99\%. Figure 13 shows the F1 scores with respect to words and input data of the learned DFA. We can see that the F1 scores are quite close to each other. And the DFA learned from the larger network is not necessary to get the best performance in terms of F1 score. Therefore, we present the experimental results on only one MNIST classifier in Section 4.

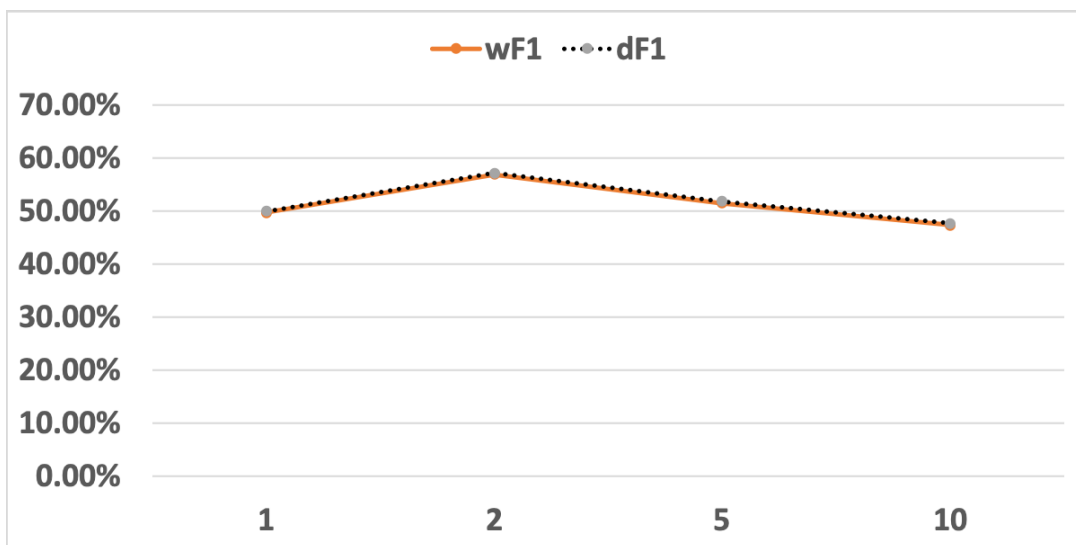

Figure 13. The F1 scores with respect to words and input data via the abstraction $2 \times 28$ 


\section{RELATED WORK}

In this section, we review some related work. Existing work on DFA extraction from neural networks targets RNNs, which was extensively explored in (Jacobsson, 2005; Wang et al., 2017).

Omlin and Giles (Omlin and Giles, 1996) proposed a global partitioning of the network state space according to $q$ equal intervals along every dimension, and then exploring the network transitions in the partitioned space. Our value abstraction adopts this partitioning, but we work on the input space, instead of the state space.

Cechin (Cechin et al., 2003) presented a approach to extract DFA using k-means and fuzzy clustering. The key idea is to classify a large sample set of reachable network state using k-means. Hou and Zhou (Hou and Zhou, 2018) proposed another approach to extract DFA from RNN using two clustering algorithms, namely LISOR-k and LISOR-x, on hidden states. There are several other work that adopted cluster analysis on state space, including k-means clustering (Zeng et al., 1993; Frasconi et al., 1996; Gori et al., 1998; Cohen et al., 2017), hierarchical clustering (Sanfeliu and Alquezar, 1994), and self-organizing maps (Tiňo and Šajda, 1995). These approaches have to access the state-vectors, while our approach is a black-box one.

Recently, Weiss et al. (Weiss et al., 2018) adopted active learning to extract automata from RNN. Our work is inspired by and similar to this, but different in the follows: (1) we target general neural network, not only RNN; (2) we consider an input is a word, rather than a symbol; (3) we use a DFA that is inferred from some training data as an abstract model for equivalent queries.

\section{CONCLUSION}

In this work, we have proposed a MAT framework to extract automata from neural networks, employing abstraction interpretation of the neural networks for answering membership and equivalence queries. We have implemented our approach in a prototype and have carried out some interesting experiments on a MNIST classifier. Through experiments, we have found that the DFA extracted from the MNIST classifier under the abstraction with the interval number 2 and the block size $1 \times 28$ performs the best. In the experiments, our resulted DFA has a better performance than the DFAs learned via the passive algorithms provided in LearnLib on the MNIST dataset.

As for future work, we may consider a better encoding such as RLE to improve the approach. We can improve the RPNI algorithm with incremental learning to reduce the learning time. We can also perform experiments on other neural network classifiers. Other models to be extracted from neural network are under consideration.

\section{REFERENCES}

Adriaans, P. W. and Jacobs, C. J. H. (2006). Using MDL for grammar induction. In 8th International Colloquium on Grammatical Inference: Algorithms and Applications (ICGI 2006), volume 4201, pages 293-306.

Aichernig, B. K., Mostowski, W., Mousavi, M. R., Tappler, M., and Taromirad, M. (2018). Model learning and model-based testing. In Machine Learning for Dynamic Software Analysis: Potentials and Limits, volume 11026, pages 74-100.

Angluin, D. (1987). Learning regular sets from queries and counterexamples. Information and Computation, 75(2):87-106.

Biggio, B., Corona, I., Maiorca, D., Nelson, B., Šrndić, N., Laskov, P., Giacinto, G., and Roli, F. (2013). Evasion attacks against machine learning at test time. In Th European Conference on Machine Learning and Knowledge Discovery in Databases, pages 387-402.

Cechin, A. L., Simon, D. R. P., and Stertz, K. (2003). State automata extraction from recurrent neural nets using k-means and fuzzy clustering. In Chilean Computer Science Society, 2003. Sccc 2003. Proceedings. International Conference of the, pages 73-78.

Cicchello, O. and Kremer, S. C. (2002). Beyond EDSM. In 6th International Colloquium on Grammatical Inference: Algorithms and Applications (ICGI 2002), volume 2484, pages 37-48.

Cohen, M., Caciularu, A., Rejwan, I., and Berant, J. (2017). Inducing regular grammars using recurrent neural networks. CoRR, abs/1710.10453.

Dupont, P. (1996). Incremental regular inference. Proceedings of the Third ICGI-96, 1147:222-237. 
Frasconi, P., Gori, M., Maggini, M., and Soda, G. (1996). Representation of finite state automata in recurrent radial basis function networks. Machine Learning, 23(1):5-32.

Fujiwara, S., Bochmann, G. V., Khendek, F., Amalou, M., and Ghedamsi, A. (1991). Test selection based on finite state models. IEEE Transactions on Software Engineering, 17(6):591-603.

Gori, M., Maggini, M., Martinelli, E., and Soda, G. (1998). Inductive inference from noisy examples using the hybrid finite state filter. IEEE Transactions on Neural Networks, 9(3):571-575.

Hou, B. and Zhou, Z. (2018). Learning with interpretable structure from rnn. CoRR, abs/1810.10708.

Howar, F., Isberner, M., Merten, M., and Steffen, B. (2012). LearnLib Tutorial: From Finite Automata to Register Interface Programs. Springer Berlin Heidelberg.

Huang, X., Kwiatkowska, M., Wang, S., and Wu, M. (2017). Safety verification of deep neural networks. In Computer Aided Verification, pages 3-29, Cham. Springer International Publishing.

Jacobsson, H. (2005). Rule extraction from recurrent neural networks: Ataxonomy and review. Neural Computation, 17(6):1223-1263.

Lecun, Y., Bengio, Y., and Hinton, G. (2015). Deep learning. Nature, 521(7553):436.

Nielson, F., Nielson, H. R., and Hankin, C. (1999). Principles of Program Analysis. Springer.

Omlin, C. W. and Giles, C. L. (1996). Extraction of rules from discrete-time recurrent neural networks. Neural Networks, 9(1):41-52.

Omlin, C. W. and Giles, C. L. (2000). Symbolic knowledge representation in recurrent neural networks: insights from theoretical models of computation. Knowledge-based neurocomputing, pages 63-116.

Oncina, J. and García, P. (1992). Inferring regular languages in polynomial updated time. Pattern Recognition And Image Analysis, pages 49-61.

Rodger, S. and Finley, T. (2006). JFLAP - An Interactive Formal Languages and Automata Package. Jones and Bartlett.

Sanfeliu, A. and Alquezar, R. (1994). Active grammatical inference: a new learning methodology. In in Shape, Structure and Pattern Recogniton, D. Dori and A. Bruckstein (eds.), World Scientific Pub, pages 191-200.

Schmidhuber, J. (2014). Deep learning in neural networks: an overview. Neural Netw, 61:85-117.

Szegedy, C., Zaremba, W., Sutskever, I., Bruna, J., Erhan, D., Goodfellow, I., and Fergus, R. (2013). Intriguing properties of neural networks. Computer Science.

Tiňo, P. and Šajda, J. (1995). Learning and extracting initial mealy automata with a modular neural network model. Neural Computation, 7(4):822-844.

Vaandrager, F. (2017). Model learning. Communications of the Acm, 60(2):86-95.

Wang, Q., Zhang, K., II, A. G. O., Xing, X., Liu, X., and Giles, C. L. (2017). An empirical evaluation of recurrent neural network rule extraction. CoRR, abs/1709.10380.

Weiss, G., Goldberg, Y., and Yahav, E. (2018). Extracting automata from recurrent neural networks using queries and counterexamples. In Proceedings of the 35th International Conference on Machine Learning, volume 80, pages 5247-5256. PMLR.

Zeng, Z., Goodman, R., and Smyth, P. (1993). Learning finite state machines with self-clustering recurrent networks. Neural Computation, 5(6):976-990.

Zenil, H. and Quiroz, F. H. (2006). On the possible computational power of the human mind. CoRR, abs/cs/0605065. 